\title{
Exploring the interactions among SNARC effect, finger counting direction and embodied cognition
}

\author{
Giulia Prete ${ }^{\text {Corresp., } 1}$, Luca Tommasi ${ }^{1}$ \\ ${ }^{1}$ Department of Psychological, Health and Territorial Sciences, "G. d'Annunzio" University of Chieti-Pescara, Chieti, Italy \\ Corresponding Author: Giulia Prete \\ Email address: giulia.prete@unich.it
}

The Spatial Numerical Association of Response Code (SNARC) is the preferential association between smaller/larger magnitudes and left/right side, respectively. Some evidence suggest a link between SNARC and a left-to-right finger counting habit. We asked 268 participants to show how they use the hands to count from 1 to 10. By means of this ecological task, $80 \%$ of the sample use first the right hand (to count from 1 to 5 ) and the majority of them use a palm-up posture. In Experiment $2(\mathrm{~N}=46)$ right-starters were asked to categorize 1-to-5 magnitudes as even or odd, using the left and right hand. Stimuli were presented both as Arabic numbers and by means of left and right hand photographs in palm-up and palm-down posture. Results confirmed the expected SNARC effect in the Arabic condition. With hand images we found that right hand responses were better for larger than for smaller magnitudes (SNARC, mainly for left hand palm-up stimuli), showing that the SNARC can be generalized to different codes. Finally, the interactions between magnitudes and left/right hand images in palm-up and palm-down posture suggest that embodied cognition can influence numerical processing. 
1

2

3

4

5

6

7 Chieti-Pescara, Chieti, Italy

8

9

10

11

12

13

14

15

16

17

18

19

20

21

22

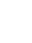

5

Chieti-Pescara, Chieti, Italy

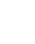

9

10

* Corresponding author:

Blocco A, Via dei Vestini 29

I-66013 Chieti, Italy

e-mail: giulia.prete@unich.it

Tel.: +39871 3554216

Fax: +39 87135542163

19 Acknowledgments

\section{Exploring the interactions among SNARC effect,}

\section{finger counting direction and embodied cognition}

Giulia Prete* and Luca Tommasi

Department of Psychological, Health and Territorial Sciences, "G. d'Annunzio" University of

20 We thank Mirko Rezzolla, who helped us in recruiting and testing participants. 


\section{Abstract}

The Spatial Numerical Association of Response Code (SNARC) is the preferential association between smaller/larger magnitudes and left/right side, respectively. Some evidence suggest a link between SNARC and a left-to-right finger counting habit. We asked 268 participants to show how they use the hands to count from 1 to 10 . By means of this ecological task, $80 \%$ of the sample use first the right hand (to count from 1 to 5) and the majority of them use a palm-up posture. In Experiment $2(\mathrm{~N}=46)$ right-starters were asked to categorize 1-to-5 magnitudes as even or odd, using the left and right hand. Stimuli were presented both as Arabic numbers and by means of left and right hand photographs in palm-up and palm-down posture. Results confirmed the expected SNARC effect in the Arabic condition. With hand images we found that right hand responses were better for larger than for smaller magnitudes (SNARC, mainly for left hand palm-up stimuli), showing that the SNARC can be generalized to different codes. Finally, the interactions between magnitudes and left/right hand images in palm-up and palm-down posture suggest that embodied cognition can influence numerical processing.

Keywords: Spatial Numerical Association of Response Code (SNARC); finger counting; magnitude; Mental Number Line (MNL); hands.

Running title: Finger counting and SNARC effect 


\section{1. Introduction}

43 1.1. The Spatial-Numerical Association of Response Codes (SNARC)

$44 \quad$ The association between numbers and space has been widely shown in decades of research

45 (for a recent review see Guida and Campitelli, 2019). For instance, in Western culture,

46 smaller/larger numbers are categorized faster with the left/right hand, respectively. This

47 association between small-left and large-right is also evident in magnitude irrelevant tasks, such

48 as parity judgments (i.e., categorizing numbers as even or odd). This automatic association is described by Dehaene, Bossini, and Giraux (1993). In this seminal study, participants were asked to categorize one-digit numbers (range: from 0 to 9) as even or odd: the results revealed that participants were faster at categorizing smaller numbers with the left hand and larger numbers with the right hand. The authors explained the SNARC effect as due to a Mental Number Line (MNL), a mental representation of quantities, organized from the leftmost to the rightmost portion of an imaginary horizontal line, in which smaller elements are positioned towards the leftmost pole and larger elements are positioned towards the rightmost pole. According to this model, the detection of a magnitude leads to the automatic placement of such a number size in a specific position along the MNL, and to the following facilitation in responding to it by using the ipsilateral hand (e.g., left hand for "leftward placed" magnitudes). Dehaene and colleagues (1993) also showed that the same number is preferentially associated with a left or right response depending on whether it is larger or smaller within a specific range, meaning that the magnitude of a number is relative to that of the specific set of numbers used (e.g., 4 and 5 are small in a 4-9 range, but they are large in a 0-5 range). This pioneering study also revealed that the SNARC is a consistent and pervasive effect, being present in both right handed and left handed individuals. 
65 However, the effect is weak in individuals educated in a right-to-left writing system (see also

66 Zebian, 2005), and thus the authors concluded that it is dependent upon a cultural and

67 educational substrate. The strength of the effect has been further confirmed in different tasks: for

68 instance, Fischer (2001) found that when participants are asked to localize the midpoint of digit

69 strings, the bisection is mislocalized either to the left or to the right with respect to the real center

70 of the strings, according to the magnitude of the digits used (e.g., leftward/rightward for "11111"

71 and "99999", respectively). Similar results were found by using number words of the same

72 length but referred to smaller or larger numbers (Calabria \& Rossetti, 2005), revealing the

73 independence of the effect from the specific code used (e.g., Arabic numbers, letters, strings).

74 More recent findings, however, suggest that culture or education do not have a causal role in the

75 development of the SNARC effect, because it has been found that a similar effect is present also

76 in non-human species (e.g., Rugani, Vallortigara, Priftis, \& Regolin, 2015; Vallortigara, 2018),

77 as well as in newborns (Di Giorgio et al., 2019).

78 The existence of an automatic SNARC effect is now unquestionable, even if it is still not clear whether it is mainly due to cultural reasons, to finger counting habits, or it is an innate bias.

\subsection{Different hypotheses on the origin of the SNARC: Mental Number Line vs finger counting}

\section{2 direction}

The most acknowledged explanation of the SNARC is the shared parietal substrate of numbers (magnitude) and space (Walsh, 2003), corresponding to the neural basis for the MNL. In support of this view, it is well-known that the parietal lobe is a region crucial for visuospatial processing, and that a parietal damage can cause the Gerstmann syndrome, involving

87 dyscalculia, agraphia, finger agnosia, and left-right confusion (Gerstmann, 1940). Moreover, a 
88 functional near-infrared spectroscopy study confirmed that the hemodynamic signature of the

89 SNARC effect is in the bilateral intraparietal sulcus and in the left angular gyrus (Cutini, Scarpa,

90 Scatturin, Dell'Acqua, \& Zorzi, 2014).

91 An alternative hypothesis has been proposed for the SNARC effect, based on the direction

92 of the finger counting habit: the "manumerical hypothesis" (Fischer \& Brugger, 2011) posits that

93 the association between magnitude and space develops during childhood, and that the cerebral

94 areas involved in finger and number processing overlap, at least partially (e.g., Thompson, Abbott, Wheaton, Syngeniotis, \& Puce, 2004). According to this hypothesis, the SNARC effect in Western culture would be due to the fact that finger counting often starts with the left hand, so that smaller numbers (from 1 to 5) are located on the left (left hand), and larger numbers (from 6 to 10) are located on the right (right hand). The hypothesis is supported by Fischer (2008), who found that the SNARC effect was strong in participants who used the left hand to start counting (left-starters), and it was absent in participants who used the right hand to start counting (rightstarters). In particular, the author carried out two tasks: Experiment 1 was aimed to designate participants as left- or right-starters, by means of a printed questionnaire containing a schematic drawing of two supine, palm-up hands (thumbs pointing outwards). Participants were invited to imagine counting with their fingers from 1 to 10 and then to write the corresponding number next to each finger on the drawing. Fischer found that, in a sample of Scottish participants, $66 \%$ were left-starters and only $34 \%$ were right-starters, and that the result was independent of participants' handedness. In Experiment 2, carried out by a subgroup of participants, the author confirmed the expected SNARC in a group of left-starters, but no reliable SNARC was found in a group of right-starters. The higher proportion of left-starters, with respect to right-starters, was 110 then replicated by Lindemann, Alipour, and Fischer (2011), who asked participants to hold out 
111 their empty hands in a supine position before finger counting, and then to remember this image

112 and to complete a computerized version of the test, selecting the matching numbers for each

113 finger shown on a computer screen. Less than the $10 \%$ of participants showed a different

114 performance between the paper-and-pencil and the computerized version of the test, and the

115 authors interpreted this result as a confirmation of the validity of the large scale paper-and-pencil

116 test used by Fischer (2008). It has to be highlighted that in the same study the authors also

117 revealed the importance of the culture on finger counting habits, with $68 \%$ of left-starters in

118 Western participants and 63.4\% of right-starters in Middle-Eastern participants. Of note, among

119 Western participants, Italians and Belgians did not reveal a significant preference for starting to

120 count with the left hand, differently from participants from the Netherlands, United Kingdom,

121 Canada, Finland, Germany and the United States (Lindemann et al., 2011). However, Lucidi and

122 Thevenot (2014) found that $26 \%$ of a sample of adults revealed a mismatch between a

123 questionnaire and an ecological task on finger counting. Similarly, Wasner, Moeller, Fischer and

124 Nuerk (2014) confirmed that finger counting is influenced by the strategy of counting used: they

125 found that $28 \%$ of participants started counting with their left hand in a spontaneous counting

126 condition, but this percentage increased to $54 \%$ in a left-to-right perceptual arrangement of

127 fingers, and it grew up to $62 \%$ in a left-to-right perceptual arrangement condition in which the

128 participant' dominant hand was busy. More recently, Cipora and colleagues (2019;

129 Supplementary materials) found a higher proportion of left- than right-starters in Russian and

130 Polish participants (Slavic language), but a higher proportion of right- than left-starters in

131 participants speaking Germanic (German, English), Uralic (Hungarian), Oghuz (Turkish),

132 Romantic (Spanish, Italian, Romanian) languages, as well as in Iranian (Farsi) participants,

133 whose reading/writing direction is from right to left. Thus, the authors revealed the effect of 
134 culture on the counting direction, with a focus on the language families. Sato and Lalain (2008)

135 found that in a sample of 117 French participants of different age groups, 69\% were right-starters

136 and just 26\% were left-starters. Di Luca et al. (2006) tested an Italian sample by using an original

137 task: the authors asked participants to identify Arabic digits by pressing one of ten keys by using

138 all of the ten fingers. They found that a right-to-left hand-digit mapping led to better performance

139 than did a left-to-right hand-digit mapping. Moreover, the authors also found that such a

140 mapping was faster than the SNARC-congruent mapping both when participants carried out the

141 task in palm-up and in palm-down postures (Di Luca, Granà, Semenza, Seron, \& Pesenti, 2006).

142 A right-to-left hand counting habit has been recently confirmed in a Polish sample by Hohol,

143 Wołoszyn, Nuerk and Cipora (2018), who exploited a modified version of the Fischer's

144 questionnaire: in particular, the authors firstly asked participants to count with their fingers from

1451 to 10 and to memorize the order, and only in a following moment they were presented with

146 schematic hands drawings on which they were asked to mark the respective numbers. Moreover,

147 in a parity judgment task during Transcranial Magnetic Stimulation (TMS), an increase in

148 amplitude of motor-evoked potentials of right-hand muscles was found during the presentation of

149 smaller rather than larger numbers (Sato, Cattaneo, Rizzolatti, \& Gallese, 2007). This result is in

150 accordance with other brain imaging evidence, showing a key role of the precentral gyrus

151 (together with the parietal areas) in both spatial encoding and number processing (Dehaene,

152 Spelke, Pinel, Stanescu, \& Tsivkin, 1999; Dehaene et al., 1996; de Jong, Van Zomeren,

153 Willemsen, \& Paans, 1996; Pesenti, Thioux, Seron, \& De Volder, 2000; Pinel et al., 1999;

154 Rueckert et al., 1996).

155 We can conclude that the two main theories about the origin of the SNARC are i) that

156 suggesting a central role of the MNL (Dehaene et al., 1993), according to which we 
157 spontaneously place smaller/larger magnitudes in the left/right side of the space, respectively,

158 and ii) the "manumerical hypothesis" (Fischer, 2008), according to which the placement of

159 smaller/larger magnitudes in a left-to-right axis would be associated to the habit to start finger

160 counting from the left hand (smaller numbers) and to proceed through the right hand (larger

161 numbers). It has been shown that in all cases the cerebral areas involved in the SNARC are the

162 same areas activated during spatial tasks, but the specific role of culture in its development is

163 still unclear.

\subsection{Exploring the SNARC in an embodied cognition framework}

The findings reviewed above lead to study the SNARC effect and the finger counting in a body-related point of view. According to the embodied cognition models, cognition, body and environment are considered as parts of an integrated system (e.g., Mahon \& Caramazza, 2008). In support of this frame, Di Luca and Pesenti (2008) showed that participants named numerical finger configurations faster when they were conform to the finger configurations usually used by the participants than when they did not. Importantly, they also found a facilitation in a magnitude judgment task, when an Arabic target stimulus was primed by an unconsciously perceived numeral finger configuration, mainly if the prime showed a canonical configuration of finger counting. The authors concluded that canonical finger counting configurations can activate number semantics in an automatic fashion. In this view, Atmaca, Sebanz, Prinz and Knoblich (2008) showed a joint SNARC effect when two participants performed a parity tasks together.

177 Moreover, by changing the position of the responding keyboard, You and colleagues (2018)

178 found that the object-based reference frame is not related to the SNARC effect, but that the position of the body of two participants tested together (egocentric coding) influenced the task. 
proceeded from thumb-to-little finger on both hands to perform a magnitude task and a parity their hands palm-down or palm-up. The authors found a unimanual SNARC effect with each with the left hand in the palm-up posture. The evidence has been ascribed to a posture-invariant structural representation of the hands, which seems to be stronger than the pure effect of the leftto-right MNL. Different results were found in a cross-modal task: Brozzoli and colleagues (2008) presented one digit number on a computer screen (visual presentation) and asked participants to detect a tactile stimulus applied either on the thumb or on the little finger. The authors found that when participants' right hand was in a palm-down posture, the performance was better when tactile stimuli were delivered to the little finger after the presentation of number 5 than number 1 . However, when participants' right hand was in a palm-up posture and tactile stimuli were delivered to the little finger, the performance was better after the presentation of number 1 than number 5 . They concluded that in case of competition between space- and bodybased representation of numbers, the human brain "prefers" the former, showing the stronger role played by the MNL in spatial cognition. Similarly, it has been shown that the SNARC disappears in the crossed hands condition (Wood, Nuerk, \& Willmes, 2006), suggesting that it is dependent upon the mental and/or physical disposition of the body in the space. A more complex pattern of results was found by Viarogue, Hubbard and Dehaene (2014), who found the expected SNARC in both uncrossed and crossed hands conditions, but only when instructions were focused on the left/right response buttons; the effect disappeared in the uncrossed condition when instructions were focused on the responding hand, as well as when the responses were 
203 given on the vertical axis (top/bottom buttons), concluding that the SNARC effect is dependent

204 upon a number of spatial reference frames, and it can be modulated by the specific experimental 205 context.

206

\subsection{SNARC effect, embodied cognition and finger counting}

Starting from the contrasting results found in different studies, revealing - in left-to-right writing systems - a population bias in starting the finger counting with the left hand (e.g., Fischer, 2008; Lindemann et al., 2011) or with the right hand (e.g., Sato \& Lalain; 2008; Hohol et al., 2018), the first aim of the present study was to assess this bias in a wide Italian sample. Moreover, due to the controversial results about the possible correspondence between paper-andpencil, computerized and ecological tasks, we asked participants to physically show how they use the fingers to count from 1 to 10 (Experiment 1; ecological task). Furthermore, we randomly selected a subgroup of these participants and administered to them a classic parity judgment task, in which they were invited to use the left or right hand to categorize even or odd numbers presented on a computer screen (Experiment 2). The second aim of the present study, in fact, was to confirm the SNARC effect assessed by means of Arabic numeral stimuli (from now on Arabic code) by using a restricted set of magnitudes (1-to-5), and to verify the possible existence of the SNARC effect (i.e., better performance with the left/right hand for smaller/larger numbers) in a sample that starts finger counting with the right hand. Specifically, Experiment 2 was mainly

222 aimed at disentangling the origin of the SNARC effect: according to the MNL (Dehaene et al., 1993), the SNARC effect should be independent from the direction of finger counting and it

224 should be based upon a spontaneous placement of lower/higher magnitudes on the left/right side 225 of the space, respectively; according to the manumerical hypothesis (Fischer \& Brugger, 2011), 
226 the SNARC effect should be due to the habit of starting counting with the left hand. Thus, both

227 of these two theories predict that participants who start finger counting from the left hand would

228 show a classic SNARC effect in a parity judgment task, but they differ in the prediction on right-

229 starters: participants who start finger counting from their right hand would show the SNARC

230 effect, if the MNL is the basis of the effect, but they would not reveal the SNARC effect if the

231 manumerical hypothesis is true. Considering these premises, only right-starters were involved in

232 Experiment 2, with the aim to verify which of the two main hypotheses on the origin of the

233 SNARC is valid. Starting from the literature about SNARC and embodied cognition (e.g., Riello

234 \& Rusconi, 2011; Sato et al., 2011), and from the evidence that hand configurations allow the

235 automatic activation of number semantics (Di Luca \& Pesenti, 2008), we also built an original

236 condition in which the same magnitudes used in the classic parity judgment task were presented,

237 but instead of the Arabic code we presented numbers as left/right hand photographs during finger

238 counting (e.g., number five showed by means of an open hand with the five fingers stretched, in

239 an ecological perspective). Images were kept as natural as possible (e.g., without removing

240 shadows) in order to make the computerized task as much "ecological" as possible. For both the

241 Arabic code and the photographed stimuli, we asked participants to carry out the same parity

242 judgment task, expecting to find a similar SNARC effect. Finally, starting from the different

243 results found depending on the postures of the participants' hands (palm-up, palm-down; e.g., Di

244 Luca et al., 2006; Riello \& Rusconi, 2011), instead of manipulating participants' hand posture,

245 we manipulated the postures of the stimuli (left and right hands were shown either palm-up or

246 palm-down). We expected that, according to the SNARC effect, for both Arabic code and hand

247 images the performance of participants would be better for smaller/larger numbers categorized

248 with the left/right hand, respectively (expecting to find a significant interaction between 
249 magnitude and responding hand). We also hypothesized that, according to an embodied

250 perspective, the photographs of a left/right hand would lead to a better performance with the

251 corresponding left/right hand used to respond (this could be revealed by the interaction between

252 the depicted hand used as stimulus and the responding hand). Finally, we also aimed at exploring

253 whether this hand-compatibility effect could be stronger for stimuli presented palm-down (as this

254 was the posture of the participants' hands during the task) or for stimuli presented palm-up (as

255 the viewed hands during a first-person finger counting). Thus our hypotheses were: i) a better

256 performance with the left/right hand for smaller/larger magnitudes (SNARC effect); ii) a stronger

257 SNARC effect when magnitudes are shown by means of left/right hand images (embodied

258 cognition); iii) a stronger SNARC by means of left/right hand mainly when stimuli are presented

259 palm-down (compatible with the hand posture held by participants during the task)."

260

261

\section{Experiment 1}

262

263

264

265

266

267

268

269

270

271

\subsection{Material and methods}

\subsubsection{Participants and procedure}

Participants were contacted by consulting a list of university students created for research

purposes. Only right-handed volunteers were contacted and the task was carried out by a final sample of 268 healthy participants (132 female and 136 male) with an age comprised between 18 and 39 years old (mean \pm standard error: $24.4 \pm 0.01$ years). Participants were tested in isolation and the task required less than 5 minutes.

The present study did not involve patients, children or animals, as well as drugs, genetic samples or invasive techniques, thus it was not subject to ethical review by the academic medical research board. Nevertheless, verbal informed consent was obtained from all participants and the 
272 experiment was conducted in accordance with the ethical standards prescribed by the Declaration 273 of Helsinki.

274 Participants were invited to enter a silent and empty room, taking care that they did not

275 have something in the hands, and to stand in front of the experimenter. The experimenter asked

276 the participant to show the way in which they usually used the fingers to count from 1 to 10 . No

277 reference was made to the hand to be used and no feedback was given to the participant. The

278 experimenter observed the finger counting made by each participant and categorized the "type of

279 action". Finger counting was categorized regarding: 1) the hand used to count from 1 to 5 and

280 from 6 to 10 (left or right); 2) the position of the hands during the counting (palm-up or palm-

281 down); 3) the order of the fingers "used" in each hand (e.g. from little finger to thumb or from

282 thumb to little finger).

283 After the finger counting, which was observed by the experimenter, participants were

284 invited to take part in different studies, after which a computerized version of the handedness

285 inventory was administered and then they were debriefed. The handedness score was measured

286 by means of the Italian version of the Edinburgh Handedness Inventory (Salmaso \& Longoni,

287 1985), in which a score of -100 corresponds to a complete left preference, and +100 corresponds

288 to a complete right preference (handedness score: $71.25 \pm 0.06$ ).

\subsection{Results}

Results are summarized in Table 1: a total of 205 participants $(76.49 \%)$ started the finger

292 counting with the right hand (from 1 to 5) and then used the left hand (from 6 to 10); 56

293

participants $(20.89 \%)$ started the finger counting with the left hand (from 1 to 5 ) and then used

294 the right hand (from 6 to 10); the remaining 7 participants (2.61\%) used the right hand to count 
295 from 1 to 5 and then re-used the same hand to count from 6 to 10 . A chi-square test showed that

296 the difference in starting hand was significant $\left(X^{2}{ }_{(1)}=86.92, p<0.001\right)$, with the majority of the

297 sample starting the finger counting by using the right hand.

298

299

300

301

302

303

304

305

306

307

308

309

310

311

312

313

314

315

316

317
Moreover, two chi-square tests were carried out to compare the orientation of the hands

within the subgroups of left- and right-starters: the significant result showed that finger counting was made preferentially in palm-up position in both the left-starters subgroup (palm-up: 52 vs palm-down: $1 ; X^{2}(1)=49.07, p<0.001$ ) and the right-starters subgroup (palm-up: 200 vs palmdown: $\left.3 ; X_{(1)}^{2}=191.18, p<0.001\right)$. Note that all participants assumed either a palm-up or a palm-down posture, without "ambiguous" postures (e.g., hands perpendicular to the ground). In order to assess the possible effect of the handedness score on the finger counting habits, the sample was divided into 3 subgroups considering the score obtained in the handedness questionnaire. Due to the fact that all participants were right-handed and that the possible handedness score was comprised between 0 (no handedness preference) and 100 (total right preference), we considered a first subsample of participants who scored less than $33.33(28.81 \pm$ 1.26; Group 1: no preference, $\mathrm{N}=5$ ), a second subsample for scores comprised between 33.34 and $66.66(56.04 \pm 0.88$; Group 2: weak right preference, $N=95)$, and the last subsample for scores higher that $66.67(81.12 \pm 0.71$; Group 3: strong right preference, $\mathrm{N}=168)$. For each subgroup a chi-square test was carried out comparing the frequency of left-starters vs rightstarters. After Bonferroni correction for multiple comparisons, the result in Group 1 was not significant, possibly due to the very low sample size of this subsample ( 2 vs $\left.3, X_{(1)}^{2}=0.02\right)$, but 
318 it was significant in Group $2\left(21\right.$ vs $\left.67, X_{(1)}^{2}=20.90, p<0.001\right)$ and in Group $3\left(33\right.$ vs $135, X^{2}{ }_{(1)}$

$319=59.50, p<0.001)$, confirming a higher proportion of right-starters in both weak and strong

320 right preference groups.

321

\section{2.3. Discussion}

323

In a sample of 268 right-handed participants we found that $79.1 \%$ starts finger counting

324 with the right hand and $20.9 \%$ starts finger counting with the left hand. The difference is

325 significant, showing that in the Italian population there is a bias to start counting with the right

326 hand. This result is in contrast with the results described by Fischer in a Scottish sample (66\%

327 left-starters vs 34\% right-starters), but it has to be noticed that in that study a pen-and-pencil

328 questionnaire was used. We can hypothesize that the left-to-right writing direction could have

329 had an influence on the results by Fischer, even if contrasting evidence has been recently

330 collected in samples from different countries by Cipora and colleagues (2019), suggesting that

331 culture has a key role in influencing finger counting direction. Our results are in line with those

332 collected in samples geographically closer to the one we tested, such as Germans (28\% left-

333 starters; Wasner et al., 2014) and French (26\% left-starters, Sato \& Lalain, 2008), supporting a

334 central role of culture on finger counting. Finally, we also found that finger counting is not

335 influenced by the degree of hand preference, due to the fact that both weak and strong right hand

336 preference groups revealed a significant prevalence of right-starters. This latter result could be

337 viewed as in line with the evidence that hand preference is not related to finger counting

338 (Fischer, 2008) even if caution is needed in this regard, due to the fact that only right-handed

339 participants were tested in the present study. 
341

342

343

344

350

351

352

353

354

355

356

357

358

359

360

361

362

363

\section{Experiment 2}

Experiment 2 was aimed at verifying the second main hypothesis of the present study, namely the possibility that even in a sample of right-starters (as the majority of the sample tested in Experiment 1) a SNARC effect can emerge by using a parity judgment task. If this effect will be found, the manumerical hypothesis (Fischer \& Brugger, 2011) can be discarded as the origin of the SNARC effect, in favor of the MNL (Dehaene et al., 1993). This hypothesis was tested by presented 1-to-5 magnitudes by means of both Arabic numbers and hands images, in order to also asses the possible effect of the embodied cognition on the SNARC effect.

\subsection{Material and methods}

\subsubsection{Participants}

A subsample of 48 participants (24 female), randomly selected from the sample of Experiment 1, carried out a computerized task in Experiment 2. Participants were right-handed, with a mean handedness score of $64.67( \pm 1.79)$, and with an age comprised between 19 and 28 years old (age: $23.21 \pm 0.29$ years). Forty-six participants were right-starters and 2 participants were left-starters (all participants counted with a palm-up posture), as emerged in Experiment 1. Participants were tested in isolation, they were unaware of the purpose of the study and took part at the task as volunteers.

\subsubsection{Stimuli}

Stimuli were constituted by images representing the following one-digit numbers: $1,2,4$ and 5. These four stimuli were shown both in the Arabic code and by means of photographs of hands. In particular, 8 photographs of a male right hand were created: the number 1 was depicted 
364 as a closed fist with the thumb finger lifted up; the number 2 was depicted as a closed fist with

365 the thumb finger and the index finger lifted up; the number 4 was depicted as an open hand with

366 all the fingers lifted up except for the thumb; and the number 5 was depicted as an open hand

367 with all of the 5 fingers lifted up. These finger configurations are those mostly used in Italy to

368 indicate the respective numbers, and previous evidence show no differences in the processing of

369 magnitudes presented as the participants' typical finger counting configuration or in a different,

370 culturally shared, finger configuration (Di Luca \& Pesenti, 2008). The four finger configurations

371 were represented both with the palm visible (palm-up) and with the knuckles visible (palm-

372 down). The eight right hand photographs were then flipped horizontally to create a set of eight

373 left hand stimuli. For the Arabic code, the number 1, 2, 4 and 5 were written in dark red (Calibri,

374 font 400). All of the stimuli (Arabic numbers and hand configurations) were presented on a gray

375 background measuring $720 \times 666$ pixels (height $x$ width), corresponding to $17.76^{\circ} \times 16.62^{\circ}$ of

376 visual angle, seen at a distance of about $57 \mathrm{~cm}$ (screen resolution: $1280 \times 768$ pixels).

377

378

379

380

381

382

383

384

385

386

387

\subsubsection{Procedure}

Each participant carried out two experimental sessions composed of 200 trials each: the 16 hand photographs (8 left hand and 8 right hand) and the 4 Arabic numbers (20 stimuli) were repeated 10 times, and they were presented in a randomized order. In each trial, after the presentation of a black fixation cross in the center of the white screen for $1 \mathrm{~s}$, a stimulus was presented in the center of the screen until the participant gave the response, then the subsequent trial started (see Figure 1). Participants were asked to categorize each stimulus as even or odd by pressing two different key of the keyboard. In one session they were asked to use the left index to press " $\mathrm{C}$ " for even numbers and to use the right index to press " $\mathrm{M}$ " for odd numbers, and in the other session they were asked to use the left index to press " $\mathrm{C}$ " for odd numbers and to use 
388 the right index to press " $\mathrm{M}$ " for even numbers. The order of the two sessions was balanced 389 among participants.

390

**********************igure 1 about here

392

Prior to the beginning of the task, written instructions were presented and participants were 394 informed that even numbers ( 2 and 4 ) and odd numbers ( 1 and 5) would be presented by means of Arabic code and finger configurations. The paradigm was controlled by means of E-Prime software (Psychology Software Tools Inc., Pittsburgh, PA), and lasted about 15 minutes. The experimental procedures were conducted in accordance with the guidelines of the Declaration of Helsinki.

\subsection{Results}

401

Statistical analyses were carried out by using the Statistica 8.0 software (StatSoft, Inc., 402 Tulsa, OK), with a significant threshold of $p<0.05$. Due to the low numbers of left-starters, which did not allow us a statistical comparison between left-starters and right-starters, the two left-starter male participants were excluded from the analysis. Data from the 46 right-starters were analyzed by means of two analyses of variance (ANOVA). In a first step, the performance 406 in the Arabic condition was analyzed by using Number (smaller: 1 and 2; larger: 4 and 5) and 407 Response hand (left, right) as within-subjects factors. In a second step, the performance for the 408 finger configuration stimuli was analyzed by using Depicted hand (left hand, right hand), Hand 409 posture (palm-up, palm-down), Number (smaller: 1 and 2; larger: 4 and 5) and Response hand

410 (responding hand: left, right) as within-subjects factors. In both analyses the performance 
411 obtained in the two sessions (different association between left/right hand and even/odd

412 response) was averaged and the Inverse Efficiency Score (IES) was used as the dependent

413 variable. IES was calculated dividing response time by the proportion of accuracy in each

414 condition, so that a lower IES corresponds to a better performance than a higher IES, in terms of

415 both accuracy and response time (e.g., Prete, Malatesta, \& Tommasi, 2017; Prete, D’Anselmo,

416 Brancucci, \& Tommasi, 2018). In a first version of both ANOVAs, Gender of participant

417 (female, male) was used as between-subjects factor, but it was not significant as main effect, nor

418 in interaction with the other factors, thus it was excluded from the analyses. The mean accuracy

419 of the sample was higher than $93 \%$ in each condition. Response times were considered only for

420 the correct responses and when they did not exceed two standard deviations from the mean of

421 each participant. In the ANOVAs, post-hoc comparisons were carried out by using Duncan tests.

422 Finally, in order to compare the performance obtained with Arabic numbers and hand

423 configurations, a series of one sample $t$-tests was carried out, consisting in a specific $t$-test in

424 which the statistical comparison is carried out between a distribution and a specific value used as

425 the reference value: the performance obtained with the "classical" Arabic numbers was used as

426 the reference value against which the performance obtained with hand configurations was

427 compared. This analysis was used because we aimed at comparing the performance in the

428 original task with hand images, to that obtained in the classical task with Arabic numbers. The

429 results of each $t$-test specifically reveal whether there is a difference in the participants'

430 performance between Arabic code (the reference value obtained with stimuli classically used to

431 investigate the SNARC effect) and hand images (a set of stimuli never used before to investigate

432 the possible embodied effect on the SNARC). Bonferroni correction was applied to correct for

433 multiple comparisons. 
In the first ANOVA (Arabic code) the main effect of Response hand was significant $\left(F_{(1,}\right.$

435

436

437

438

439

440

441

442

443

444

445

446

447

448

449

450

451

452

453

454

455

456

46) $\left.=5.98, M S E=1595, p=0.019, \eta_{p}^{2}=0.12\right)$, showing a better performance with the right hand $(594.64 \pm 16.48)$ than with the left hand (609.04 \pm 16.79$)$. Importantly, the interaction between

Number and Response hand was significant $\left(F_{(1,46)}=96.80, M S E=2539, p<0.001, \eta_{p}{ }^{2}=0.68\right)$.

Post-hoc tests confirmed a strong SNARC effect: the performance was better for larger numbers categorized with the right hand than with the left hand, and it was better for smaller numbers categorized with the left than with the right hand; left hand responses were better for smaller than larger numbers, and right hand responses were better for larger than for smaller numbers (all comparisons: $p<0.001$; Figure 2).

(1)

(1)

$* * * * * * * * * * * * * * * * * * * * *$ Figure 2 about here

(1)

In the second ANOVA (finger code) the main effect of Number was significant $\left(F_{(1,46)}=\right.$ $\left.22.28, M S E=4766, p<0.001, \eta_{p}^{2}=0.33\right)$, revealing a better performance for larger $(609.53 \pm$ $16.95)$ than for smaller numbers $(633.55 \pm 15.47)$. The main effect of Hand posture was significant $\left(F_{(1,46)}=5.59, M S E=1450, p=0.022, \eta_{p}{ }^{2}=0.11\right)$ : the performance was better for hands depicted palm-down $(618.23 \pm 15.54)$ than palm-up $(624.86 \pm 16.89)$, confirming the effect of the embodied perspective (participants carrying out the task with their hands on the keyboard, thus in a palm-up posture). Number and Response hand significantly interacted $\left(F_{(1,46)}\right.$ $=6.02, M S E=2847, p=0.018, \eta_{p}^{2}=0.12$; Figure 3): post-hoc tests showed that the performance was better for larger than smaller numbers with both the left $(p=0.013)$ and the right hand $(p<$ 0.001). Importantly, for larger numbers the performance was better with the right hand than with the left hand $(p=0.011)$, thus confirming - at least partially - the expected SNARC effect. 

images were palm-up than palm-down $(p=0.027)$, whereas for smaller numbers it was better when images were palm-down than palm-up $(p<0.001)$; for palm-up images the performance was better for larger than for smaller numbers $(p<0.001)$. $\left.=17.17, M S E=2229, p<0.001, \eta_{p}{ }^{2}=0.28\right):$ when left hands were presented, the performance was better for larger numbers categorized with the right response hand than with the left response hand $(p=0.002)$ and for smaller numbers categorized with the left than with the right hand $(p=0.003)$; moreover for these stimuli the performance was better with the right hand for larger than for smaller numbers $(p<0.001)$, whereas no significant difference was found

471 between smaller and larger numbers categorized with the left hand $(p=0.88)$. When stimuli

472 represented a right hand, the performance was better for smaller numbers categorized with the right response hand than with the left response hand $(p=0.040)$, and it was better for larger than

474 for smaller numbers categorized with both the left $(p<0.001)$ and the right response hand $(p=$ the left than the right hand $(p=0.006)$, whereas right-hand responses for smaller numbers were better when stimuli represented the right than the left hand $(p=0.024)$. 
480 response hand, the performance was better for larger numbers shown by means of palm-up than

481 palm-down hands ( $p=0.002)$, whereas it was better for smaller numbers shown by means of

482 palm-down than palm-up hands $(p<0.001)$. For palm-up hand images, the performance was

483 better for larger numbers categorized with the right than with the left response hand $(p=0.001)$,

484 and it was better for smaller numbers categorized with the left than with the right response hand

$485(p=0.003)$. Responses for palm-up stimuli were better for larger than for smaller numbers with

486 both the left $(p=0.016)$ and the right response hand $(p<0.001)$.

487 Finally, the four-way interaction among Depicted hand, Hand posture, Number and

488 Response hand was significant $\left(F_{(1,45)}=17.89, M S E=1872, p<0.001, \eta_{p}{ }^{2}=0.28\right)$. To better

489 understand the interaction, two ANOVAs were carried out considering palm-up stimuli and

490 palm-down stimuli separately (see Figure 4). Results showed that for palm-down stimuli the

491 interaction among Depicted hand, Number and Response hand was not significant $\left(F_{(1,45)}=0.06\right.$,

$492 M S E=1403, p=0.812)$, whereas the same interaction was significant for palm-up stimuli $\left(F_{(1,}\right.$

$\left.493{ }_{45)}=26.58, M S E=2698, p<0.001, \eta_{p}{ }^{2}=0.37\right)$. Post-hoc tests showed that for left hand images

494 the performance was better when smaller numbers were categorized with the left than with the

495 right response hand $(p<0.001)$, and it was better when larger numbers were categorized with the

496 right than with the left response hand $(p<0.001)$. Right-hand images were better categorized

497 when stimuli represented larger than smaller numbers, with both the left $(p<0.001)$ and the right

498 response hand ( $p=0.001)$, whereas for left-hand images this was true only for right hand

499 responses $(p<0.001)$. Right hand responses for larger numbers were better when stimuli

500 represented a left than a right hand $(p=0.009)$, and left-hand responses for smaller numbers

501 were better for left than for right-hand images $(p=0.002)$.

502 
504

505

In order to further assess the possible difference in the processing of magnitudes presented

506

507

508

509

510

511

512

513

514

515

516

517

518

519

520

521

522

523

524

525

by means of Arabic code and hand images, a series of exact $t$-tests were carried out. Specifically, IES obtained in each of the four conditions of hand images (left hand and right hand images shown palm-up and palm-down) were compared to the mean IES obtained in the Arabic code condition, for smaller/larger numbers, categorized with the left/right hand, separately (see Figure 5). After Bonferroni correction for multiple comparisons, the results revealed that the performance was better for the Arabic code than for the hand images when larger magnitudes were categorized with the right response hand (palm-up right-hand image: $t_{(45)}=3.36, p=0.006$; palm-down right-hand image: $t_{(45)}=3.18, p=0.011$; palm-down left-hand image: $t_{(45)}=4.46, p<$ 0.001), and when smaller magnitudes were categorized with the left hand (i.e., SNARC effect), except for left hand images presented palm-up (palm-up right-hand image: $t_{(45)}=5.63, p<0.001$; palm-down right-hand image: $t_{(45)}=3.81, p=0.002$; palm-down left-hand image: $t_{(45)}=3.64, p=$ 0.003). No significant difference emerged between Arabic code and hand images either for larger magnitudes categorized with the left hand, nor for smaller magnitudes categorized with the right hand (for both left and right hand images, presented palm-up and palm-down).

\section{Discussion}

The present study was aimed at assessing the finger counting direction in a Western sample (Experiment 1) and at investigating the possible relationship between finger counting and the 
526 SNARC effect (Experiment 2). Experiment 1 showed a strong population bias in finger counting

527 direction: almost the $80 \%$ of the sample started finger counting with the right hand (right-

528 starters). This result is in accordance with those described by Sato and Lalain (2008) and more

529 recently by Hohol and colleagues (2018). In this frame it has to be highlighted that a left-starting

530 bias has been reported in other studies (e.g., Fischer, 2008; Lindemann et al., 2011), by means of

531 paper-and-pencil test and online surveys. We decided to exploit an ecological test of finger

532 counting, starting from the evidence of the validity of this method in the investigation of the

533 counting direction, also shown by a test-rest analysis carried out by Hohol et al. (2018). We

534 hypothesize that the different methods used to investigate finger counting (paper-and-pencil test,

535 ecological finger counting) could be one of the reasons for the opposite patterns of results found

536 in different studies, together with the role of the culture which seems to influence the finger

537 counting habit at a population level (see Lindemann et al., 2014; Cipora et al., 2019).

538 Specifically, the need to write down the direction of finger counting on a drawing depicting the

539 two hands can be influenced by the left-to-right writing direction in the Western culture (the

540 same holds true in the case of selecting a response from a pull-down menu). In this perspective,

541 the left-to-right enumeration could be attributable to the habit of writing from left to right. On the

542 other hand, the request to show how the fingers are used to count from 1 to 10 would be

543 independent from the writing direction, and it could be more indicative of the real finger

544 counting habits.

545 In Experiment 2 we confirmed the expected SNARC effect with Arabic code stimuli in a

546 sample of participants who started finger counting with the right hand and in palm-up posture:

547 the performance was better for smaller/larger numbers categorized (as even or odd) with the

548 left/right hand, respectively (e.g., Dehaene et al., 1993; Macnamara et al., 2018). It should be 
549 underlined that the SNARC effect was significant, even if the magnitude range used was very

550 small (1-to-5), showing that such a bias is really strong and it manifests itself also with a limited

551 set of magnitudes. Moreover, we also found an overall better performance with the right than

552 with the left hand (disregarding the magnitude), possibly attributable to the fact that all

553 participants were right-handers.

554 Our results show the SNARC effect in a sample of right-starters, in contrast with the 555 expectation based on the "manumerical hypothesis" (Fischer \& Brugger, 2011). According to 556 this hypothesis, in fact, we should expect that right-starters show a better performance with the

557 right hand for smaller numbers and with the left hand for larger numbers, assuming that the

558 SNARC effect is linked to the finger counting direction. Our results do not confirm this

559 hypothesis, showing a strong SNARC effect even in a sample of right-starting participants. We

560 can conclude that the SNARC effect is based upon a MNL instead of being dependent upon

561 finger counting direction. Due to the fact that the present study was aimed at disentangling these

562 two hypotheses (MNL and manumerical hypothesis), only right-starting participants were tested

563 in Experiment 2. We found a significant SNARC effect even in a sample of right-starters,

564 showing a relationship between finger counting and SNARC, but a direct comparison between

565 left-starters and right-starters performance is needed in order to conclude the possible causal role

566 of finger counting on the SNARC. In this regard it has to be highlighted that Cipora et al. (2019)

567 recently found that reading/writing direction and language families have a crucial role on finger

568 counting direction. From these premises, future studies should compare the performance of right-

569 starters with that of left-starters, and cross-cultural studies should be carried out, in order to

570 further investigate the interaction between finger counting direction and culture on the SNARC

571 effect. 
573 SNARC effect is present when making numerical judgments on hand configurations. To assess

574 this, we presented photographs of left and right hands in palm-up and palm-down postures,

575 showing 1-to-5 magnitudes with fingers. It has to be highlighted that Di Luca and Pesenti (2008)

576 showed the absence of differences in the processing of magnitudes presented by means of finger

577 configurations, when these magnitudes were presented either as the participants' typical finger

578 counting configuration (e.g., number four: all fingers extended except the little finger which is

579 closed on the palm) or as "finger-montring" (the most shared finger configuration used in a

580 culture to refer to a specific number; e.g., number four: all fingers extended except the thumb

581 which is closed on the palm). The authors concluded that both of these finger configurations are

582 stored in long-term memory and are associated with the respective magnitudes. Starting from this

583 evidence, in Experiment 2 we used the "finger-montring" configurations, even if they could be

584 different from the finger counting configurations found in Experiment 1.

585 Our results confirmed the SNARC effect also when stimuli were hand images, at least

586 partially: the performance was better for larger magnitudes categorized with the right than with

587 the left hand, confirming the independence of the SNARC effect from a specific code. This

588 evidence is in accordance with previous results (Nuerk, Wood, \& Willmes, 2005) showing that

589 the SNARC effect withstands to different magnitude notations (Arabic numeral, visual number

590 word, visual dice pattern), as well as to different perceptual modalities (visual and auditory

591 number words). Moreover, we also explored the possible effect of the hand images presented as

592 stimuli (left hand, right hand) as well as the effect of the hand posture (palm-up and palm-down),

593 and we found a better performance for smaller/larger magnitude categorized with the left/right

594 hand (SNARC effect) when stimuli were left hand images. We also found that in palm-up 
595 condition, the performance of the participants was better for left hand images than for right hand

596 images when smaller/larger magnitudes were categorized with the left/right hand, respectively.

597 These results seem to suggest that a SNARC effect can be found with hand images, mainly when

598 stimuli represent left hands, in palm-up posture, suggesting a kind of interaction between the

599 MNL and hand representation: we could speculate that also these results can be intended as

600 linked to the MNL, in which all of the magnitudes presented here (from 1 to 5) could be placed

601 on the leftmost portion of the line, possibly being considered as "small" numbers, thus

602 privileging a left hand representation. Accordingly, the results of the $t$-tests further confirm that

603 the SNARC effect is stronger for magnitudes presented in the Arabic code than by means of

604 hand images: the performance of participants was better in categorizing magnitudes in Arabic

605 than in finger code in the conditions congruent with the SNARC effect (smaller magnitudes

606 categorized with the left hand and larger magnitudes categorized with the right hand) and they

607 did not differ in the conditions incongruent with the SNARC effect (smaller magnitudes

608 categorized with the right hand and larger magnitudes categorized with the left hand). These

609 significant differences were present for right hand images shown palm-up and palm-down, as

610 well as for left hand images shown palm-down. The difference between the two codes was not

611 significant for palm-up left hand images, confirming that the task benefits from the same

612 facilitation (SNARC) when stimuli are in Arabic code and left hand palm-up configurations. In

613 other words, we found that the parity judgment task required the same cognitive effort when

614 magnitudes were presented either in Arabic code or by means of hand configurations, in the

615 conditions incongruent with the SNARC effect. Nevertheless, in the SNARC-congruent

616 conditions the performance was better for Arabic code than for hand images, confirming that the

617 Arabic code is the preferential code for magnitude processing, except for magnitudes presented 
618 by means of left hand palm-up images. This pattern of results shows that left hand palm-up

619 images facilitate the magnitude processing in the same way as the Arabic code, in accordance

620 with the spatial numerical association, and we speculate that this effect could be linked to a

621 possible interaction between the SNARC effect and the left-to-right MNL. In this frame, the

622 MNL could explain a left-hand preference for 1-to-5 magnitudes, even if it does not correspond

623 to the real finger counting habit. However caution is needed in this regard, due to the evidence

624 that the magnitude of the stimuli presented seems to be mentally categorized as small or large in

625 accordance with the specific range of magnitudes used (Dehaene et al., 1993).

626 For smaller magnitudes we also found a hand compatibility effect: left-handed responses

627 were better for left than right hand images, and right-handed responses were better for right than

628 left hand images. This pattern of results confirms a body-related processing, for which stimuli

629 representing one hand are better categorized with the corresponding response hand. The fact that

630 the performance of participants was overall better when hand images were shown as palm-down

631 can be associated to the specific hand postures of the participants during the task (with the finger

632 on a keyboard, i.e. palm-down). In this perspective we can conclude that embodied cognition

633 extends to hands representation and it can modulate the responses of participants in a task not

634 requiring an explicit representation of the body posture (see Di Luca et al., 2006; Riello \&

635 Rusconi, 2011). However, the interaction revealing that the palm-down configuration enhanced

636 the performance only for larger magnitudes (smaller magnitudes being better processed when

637 presented palm-up), prevent us to support this hypothesis conclusively.

638 We also found that when stimuli were hand images, the performance was better for larger

639 than for smaller magnitudes, with both responding hands, an evidence not found in the Arabic

640 code. This evidence shows that different codes are processed in different ways, and future studies 
641 are needed to verify which kinds of codes allow magnitude representations to interact with the

642 MNL. Finally, no effect of participant gender was found with both Arabic code and hands

643 images, suggesting that females and males do not differ in this task. Since hands images were

644 created from a male hand photograph one could expect a better performance by male than female

645 participants, due to an own-gender bias (e.g., Prete et al., 2016), but the absence of significant

646 differences between female and male participants seems to disconfirm such a bias in the parity

647 judgment task. Nevertheless, future studies in which both female and male hands photographs

648 are presented will definitively disentangle the possible gender difference in this domain.

649

\section{5. Conclusions}

We can conclude that the right-to-left hand counting direction, widely found in an Italian sample by means of an ecological task, does not prevent the occurrence of a SNARC effect: this expected effect was confirmed in a sample who started counting by using the right hand. This is the most crucial result of the present study, supporting that finger counting habits and the mental number line are different processes. The SNARC effect is present not only for Arabic code numerical stimuli, but it can be detected also when a different code is used, such as finger configurations corresponding to 1-to-5 magnitudes. Finally, our results also suggest that all of these effects could be interpreted in the light of embodied cognition, but - due to the multiple interactions found - further studies are needed to disentangle to which extent body representations can influence numerical cognition.

661

662 Compliance with Ethical Standards:

\section{Conflict of interest}


664 The authors declare that they have no conflict of interest.

665

\section{Ethical approval}

667 All procedures performed in the 2 studies described were in accordance with the ethical

668 standards of the institutional research committee and with the 1964 Helsinki declaration and its

669 later amendments.

670

671 


\section{References}

673 Atmaca, S., Sebanz, N., Prinz, W., Knoblich, G., 2008. Action co-representation: the joint 674 SNARC effect. Soc. Neurosci., 3(3-4), 410-420. doi: 10.1080/17470910801900908

675 Brozzoli, C., Ishihara, M., Göbel, S.M., Salemme, R., Rossetti, Y., Farnè, A., 2008. Touch 676 perception reveals the dominance of spatial over digital representation of numbers. Proc. Natl. Acad. Sci., 105(14), 5644-5648. doi: 10.1073_pnas.0708414105

678 Calabria, M., Rossetti, Y., 2005. Interference between number processing and line bisection: a 679 methodology. Neuropsychologia, 43(5), 779-783. doi:

$680 \quad$ 10.1016/j.neuropsychologia.2004.06.027

681 Cutini, S., Scarpa, F., Scatturin, P., Dell'Acqua, R., Zorzi, M., 2012. Number-space interactions 682 in the human parietal cortex: Enlightening the SNARC effect with functional near-infrared 683 spectroscopy. Cereb. Cortex, 24(2), 444-451. doi: 10.1093/cercor/bhs321

684

685

686

687

688

689

690

691

692

693

694

De Jong, B.M., Van Zomeren, A.H., Willemsen, A.T.M., Paans, A.M.J., 1996. Brain activity related to serial cognitive performance resembles circuitry of higher order motor control. Exp. Brain Res., 109(1), 136-140. doi: 10.1007/BF00228634

Dehaene, S., Bossini, S., Giraux, P., 1993. The mental representation of parity and number magnitude. J. Exp. Psychol., 122: 371-396. doi: 10.1037//0096-3445.122.3.371

Dehaene, S., Spelke, E., Pinel, P., Stanescu, R., Tsivkin, S., 1999. Sources of mathematical thinking: Behavioral and brain-imaging evidence. Science, 284(5416), 970-974. doi: $10.1126 /$ science. 284.5416 .970

Dehaene, S., Tzourio, N., Frak, V., Raynaud, L., Cohen, L., Mehler, J., Mazoyer, B., 1996. Cerebral activations during number multiplication and comparison: a PET study. Neuropsychologia, 34(11), 1097-1106. doi: 10.1016/0028-3932(96)00027-9 
695 Di Giorgio, E., Lunghi, M., Rugani, R., Regolin, L., Dalla Barba, B., Vallortigara, G., Simion, 696 F., 2019. A mental number line in human newborns. Dev. Sci., e12801. doi:

697

698

699

700

701

702

703

704

705

706

707

708

709

710

711

712

713

714

715

716

717

10.1111/desc. 12801

Di Luca, S., Granà, A., Semenza, C., Seron, X., Pesenti, M., 2006. Finger-digit compatibility in Arabic numeral processing. Q. J. Exp. Psychol., 59(9), 1648-1663. doi:

$10.1080 / 17470210500256839$

Di Luca, S., Pesenti, M., 2008. Masked priming effect with canonical finger numeral configurations. Exp. Brain Res., 185(1), 27-39. doi: 10.1007/s00221-007-1132-8

Fischer, M.H., 2001. Number processing induces spatial performance biases. Neurology, 57(5), 822-826.

Fischer, M.H., 2008. Finger counting habits modulate spatial-numerical associations. Cortex, 44(4), 386-392. doi: 10.1016/j.cortex.2007.08.004

Fischer, M.H., Brugger, P., 2011. When digits help digits: spatial-numerical associations point to finger counting as prime example of embodied cognition. Front. Psychol., 2, 260. doi: 10.3389/fpsyg.2011.00260

Gerstmann, J., 1940. Syndrome of finger agnosia, disorientation for right and left, agraphia and acalculia: local diagnostic value. Arch. Neurol. Psychiatry, 44(2), 398-408.

Guida, A., \& Campitelli, G. (2019). Explaining the SPoARC and SNARC effects with knowledge structures: An expertise account. Psychonomic bulletin \& review, 1-18. doi: 10.3758/s13423-019-01582-0.

Hohol, M., Wołoszyn, K., Nuerk, H.C., Cipora, K., 2018. A large-scale survey on finger counting routines, their temporal stability and flexibility in educated adults. PeerJ, 6 , e5878. doi: 10.7717/peerj.5878 
718 Lindemann, O., Alipour, A., Fischer, M.H., 2011. Finger counting habits in middle eastern and

719 western individuals: an online survey. J. Cross Cult. Psychol., 42(4), 566-578. doi:

720 $10.1177 / 0022022111406254$

721

722

723

724

725

726

727

728

729

730

731

732

733

734

735

736

737

738

Lucidi, A., Thevenot, C., 2014. Do not count on me to imagine how I act: behavior contradicts questionnaire responses in the assessment of finger counting habits. Behav. Res. Methods, 46(4), 1079-1087. doi: 10.3758/s13428-014-0447-1

Macnamara, A., Keage, H.A., Loetscher, T., 2018. Mapping of non-numerical domains on space: a systematic review and meta-analysis. Exp. Brain Res., 1-12. doi: 10.1007/s00221-0175154-6

Mahon, B.Z., Caramazza, A., 2008. A critical look at the embodied cognition hypothesis and a new proposal for grounding conceptual content. J. Physiol. Paris, 102 (1-3), 59-70. doi: 10.1016/j.jphysparis.2008.03.004

Nuerk, H.C., Wood, G., Willmes, K., 2005. The universal SNARC effect: the association between number magnitude and space is amodal. Exp. Psychol., 52(3), 187-194. doi: $10.1027 / 1618-3169.52 .3 .187$

Pesenti, M., Thioux, M., Seron, X., Volder, A.D., 2000. Neuroanatomical substrates of Arabic number processing, numerical comparison, and simple addition: A PET study. J. Cogn. Neurosci., 12(3), 461-479. doi: 10.1162/089892900562273

Pinel, P., Le Clec'H, G., Van de Moortele, P.F., Naccache, L., Le Bihan, D., Dehaene, S., 1999. Event-related fMRI analysis of the cerebral circuit for number comparison. Neuroreport, 10(7), 1473-1479. 
739 Prete, G., D’Anselmo, A., Brancucci, A., Tommasi, L., 2018. Evidence of a Right Ear

740 Advantage in the absence of auditory targets. Sci. Rep., 8(1), 15569. doi: 10.1038/s41598-

741 018-34086-3

742 Prete, G., Fabri, M., Foschi, N., Tommasi, L., 2016. Face gender categorization and hemispheric asymmetries: contrasting evidence from connected and disconnected brains. Neuroscience, 339, 210-218. doi: 10.1016/j.neuroscience.2016.10.021

Prete, G., Malatesta, G., \& Tommasi, L., 2017. Facial gender and hemispheric asymmetries: A

747

748

749

750

751

752

753

754

755

756

757

758

759

760

761 hf-tRNS study. Brain Stimul., 10(6), 1145-1147. doi: 10.1016/j.brs.2017.08.002

Riello, M., Rusconi, E., 2011. Unimanual SNARC effect: hand matters. Front. Psychol., 2, 372. doi: 10.3389/fpsyg.2011.00372

Rueckert, L., Lange, N., Partiot, A., Appollonio, I., Litvan, I., Le Bihan, D., Grafman, J., 1996. Visualizing cortical activation during mental calculation with functional MRI. Neuroimage, 3(2), 97-103.

Rugani, R., Vallortigara, G., Priftis, K., Regolin, L., 2015. Number-space mapping in the newborn chick resembles humans' mental number line. Science, 347(6221), 534-536. doi: 10.1126/science.aaa1379

Salmaso, D., Longoni, A.M., 1985. Problems in the assessment of hand preference. Cortex, 21(4), 533-549.

Sato, M., Lalain, M., 2008. On the relationship between handedness and hand-digit mapping in finger counting. Cortex, 44(4), 393-399. doi: 10.1016/j.cortex.2007.08.005

Sato, M., Cattaneo, L., Rizzolatti, G., Gallese, V., 2007. Numbers within our hands: modulation of corticospinal excitability of hand muscles during numerical judgment. J. Cogn. Neurosci., 19(4), 684-693. doi: 10.1162/jocn.2007.19.4.684 
762 Thompson, J.C., Abbott, D.F., Wheaton, K.J., Syngeniotis, A., Puce, A., 2004. Digit

763 representation is more than just hand waving. Cogn. Brain Res., 21(3), 412-417. doi:

$764 \quad$ 10.1016/j.cogbrainres.2004.07.001

765 Vallortigara, G. (2018). Comparative cognition of number and space: the case of geometry and 766 of the mental number line. Philos. T. R. Soc. B: Biol. Sci., 373(1740), 20170120. doi:

$767 \quad 10.1098 /$ rstb. 2017.0120

768 Viarouge, A., Hubbard, E.M., Dehaene, S., 2014. The organization of spatial reference frames 769 involved in the SNARC effect. Q. J. Exp. Psychol., 67(8), 1484-1499. doi:

$770 \quad 10.1080 / 17470218.2014 .897358$

771 Walsh, V., 2003. A theory of magnitude: common cortical metrics of time, space and 772 quantity. Trends Cogn. Sci., 7(11), 483-488. doi: 10.1016/j.tics.2003.09.002

773 Wasner, M., Moeller, K., Fischer, M.H., Nuerk, H.C., 2014. Aspects of situated cognition in 774 embodied numerosity: the case of finger counting. Cogn. Process., 15(3), 317-328. doi: 10.1007/s10339-014-0599-z

Wood, G., Nuerk, H.C., Willmes, K., 2006. Crossed Hands and the Snarc Effect: Afailure to Replicate Dehaene, Bossini and Giraux (1993). Cortex, 42(8), 1069-1079. doi:

You, X., Zhang, Y., Zhu, R., Guo, Y., 2018. Embodied interaction priority: Other's body part $10.1016 / \mathrm{S} 0010-9452(08) 70219-3$

Zebian, S., 2005. Linkages between number concepts, spatial thinking, and directionality of writing: the SNARC effect and the reverse SNARC effect in English and Arabi monoliterates, biliterates, and illiterate Arabic speakers. J. Cognit. Cult., 5(1), 165-190. 


\section{Table caption:}

788 Tab 1. Pattern of finger counting from 1 to 10 in Experiment 1: frequencies (column N) and 789 percentages (column \%). The first column indicates the hand posture: UP for "palm-up", DW for 790 "palm-down". Letter pairs represent the hand (L for "left"; $\mathrm{R}$ for "right") and the finger used to 791 indicate the number at the top of the column (T: thumb, I: index, M: middle, R: ring, P: pinkie)

Figure captions:

795

796

797

798

799

800

801

802

803

804

805

806

807

808

809

Fig 1. Example of a trial in Experiment 2: after a fixation cross presented for $1 \mathrm{~s}$, a stimulus was presented in the center of the screen until the participant categorized it as even or odd: in the figure, the magnitude 2 is presented in Arabic code (panel A) and in finger code (panel B: lefthand stimulus in palm-up posture)

Fig 2. Interaction between Response hand and Number: the interaction is referred to the first ANOVA (Arabic code; Hand: left, right; Number: Smaller $=1$ and 2, Larger $=4$ and 5). Bars represent standard errors. Asterisks show the significant comparisons

Fig 3. Interaction between Response hand and Number: the interaction is referred to the second ANOVA (finger code; Hand: left, right; Number: Smaller $=1$ and 2, Larger $=4$ and 5).

Bars represent standard errors. Asterisks show the significant comparisons

Fig 4. Interaction among Depicted hand, Response hand and Number: the graph shows the results obtained in two separate ANOVAs carried out for Palm-down images (panel A, no 
810 significant interaction) and Palm-up images (panel B, significant interaction), separately. Bars

811 represent standard errors. Asterisks show the significant comparisons

812

813 Fig 5. Performance of participants in Experiment 2: the gray columns show the performance

814 with hand images (bars represent standard errors). Boxes in the first row represent the SNARC-

815 congruent conditions (left: smaller magnitudes categorized with the left hand; right: larger

816 magnitudes categorized with the right hand), boxes in the second row represent the SNARC-

817 incongruent conditions (left: larger magnitudes categorized with the left hand; right: smaller

818 magnitudes categorized with the right hand). The horizontal lines in each box represent the mean

819 performance with Arabic code numbers (continuous line shows the significant difference, dotted

820 line shows the non-significant difference between Arabic code and finger code)

821

822 
Figure 1

Example of a trial in Experiment 2

Example of a trial in Experiment 2: after a fixation cross presented for $1 \mathrm{~s}$, a stimulus was presented in the center of the screen until the participant categorized it as even or odd: in the figure, the magnitude 2 is presented in Arabic code (panel A) and in finger code (panel B: left-hand stimulus in palm-up posture)

A) Arabic code

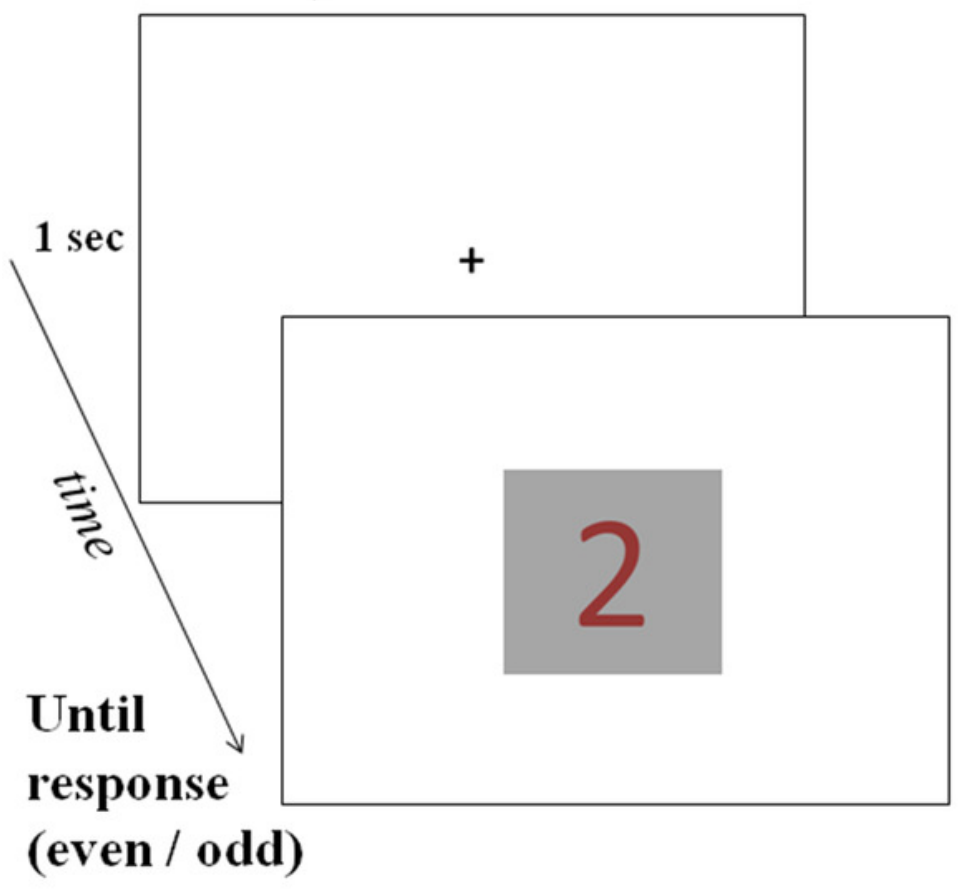

B) Finger code

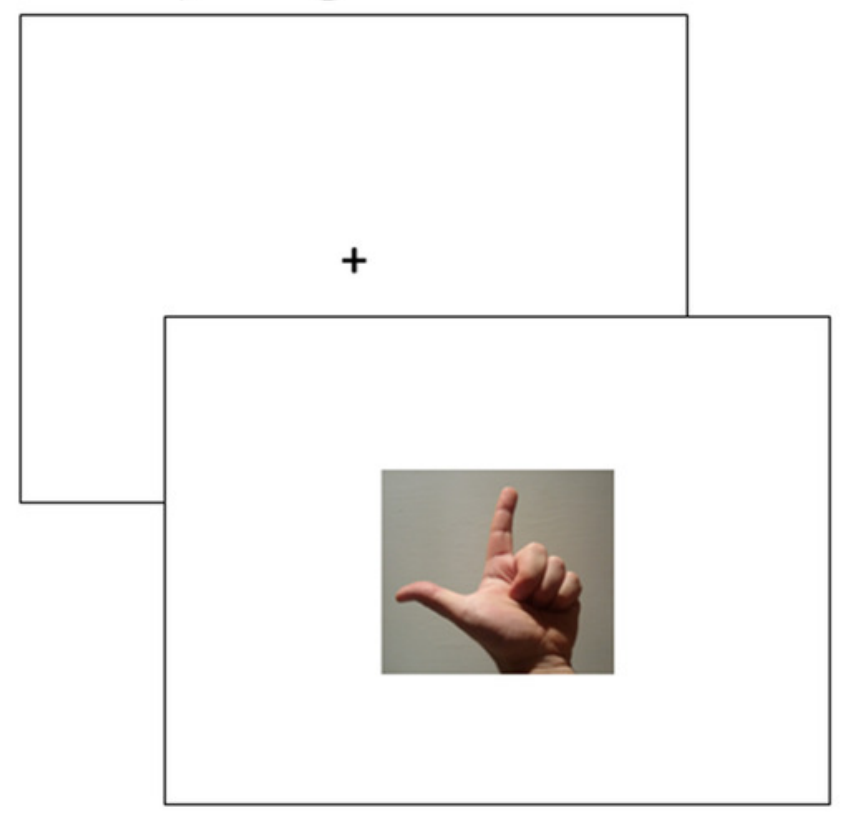




\section{Figure 2}

Interaction between Response hand and Number(Smaller: 1, 2; Larger: 4, 5)

The interaction is referred to the first ANOVA (Arabic code; Hand: left, right; Number: Smaller $=1$ and 2, Larger $=4$ and 5). Bars represent standard errors. Asterisks show the significant comparisons

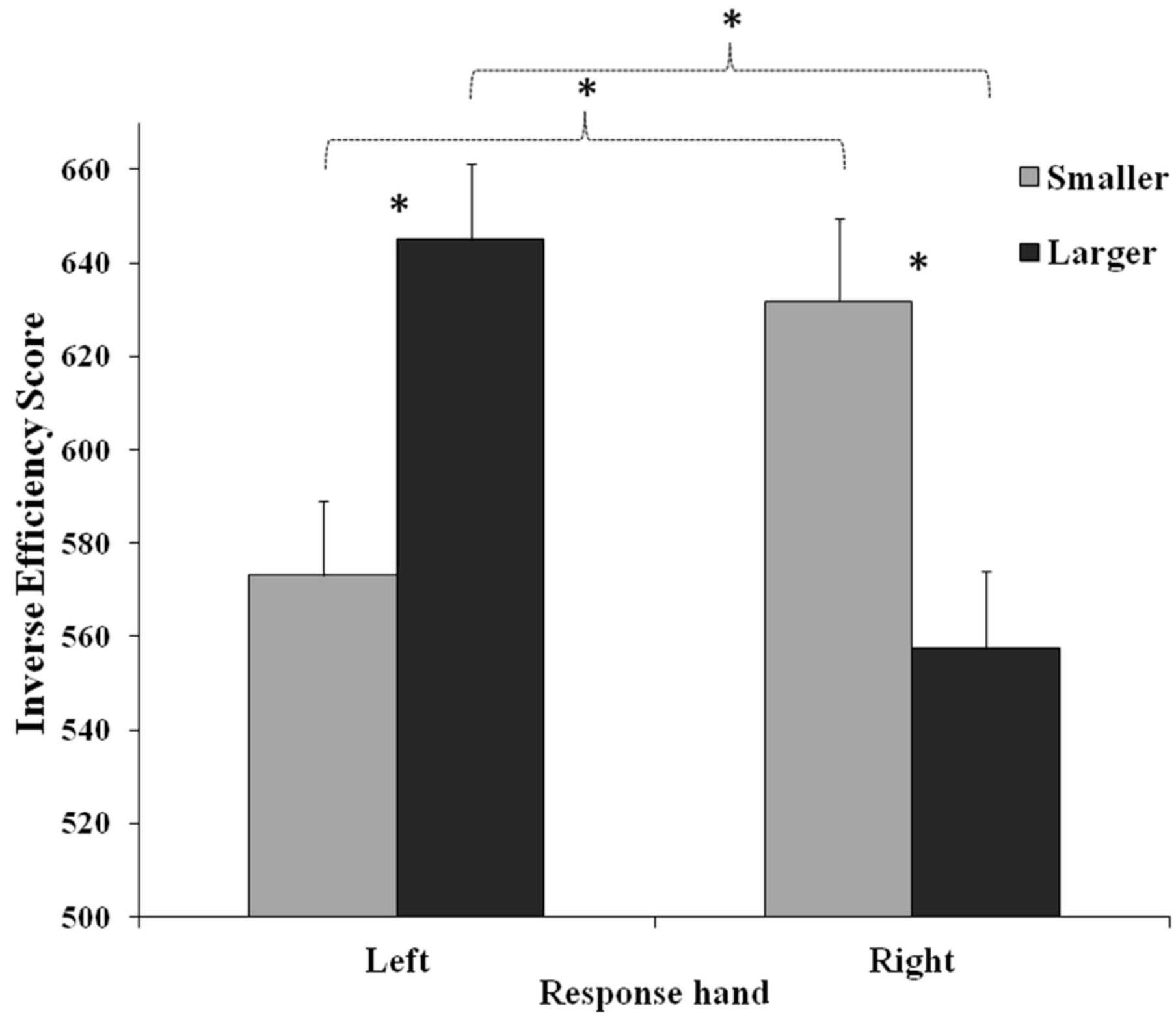




\section{Figure 3}

Interaction between Response hand and Number (Smaller: 1, 2; Larger: 4, 5)

The interaction is referred to the second ANOVA (finger code; Hand: left, right; Number:

Smaller $=1$ and 2, Larger $=4$ and 5). Bars represent standard errors. Asterisks show the significant comparisons.

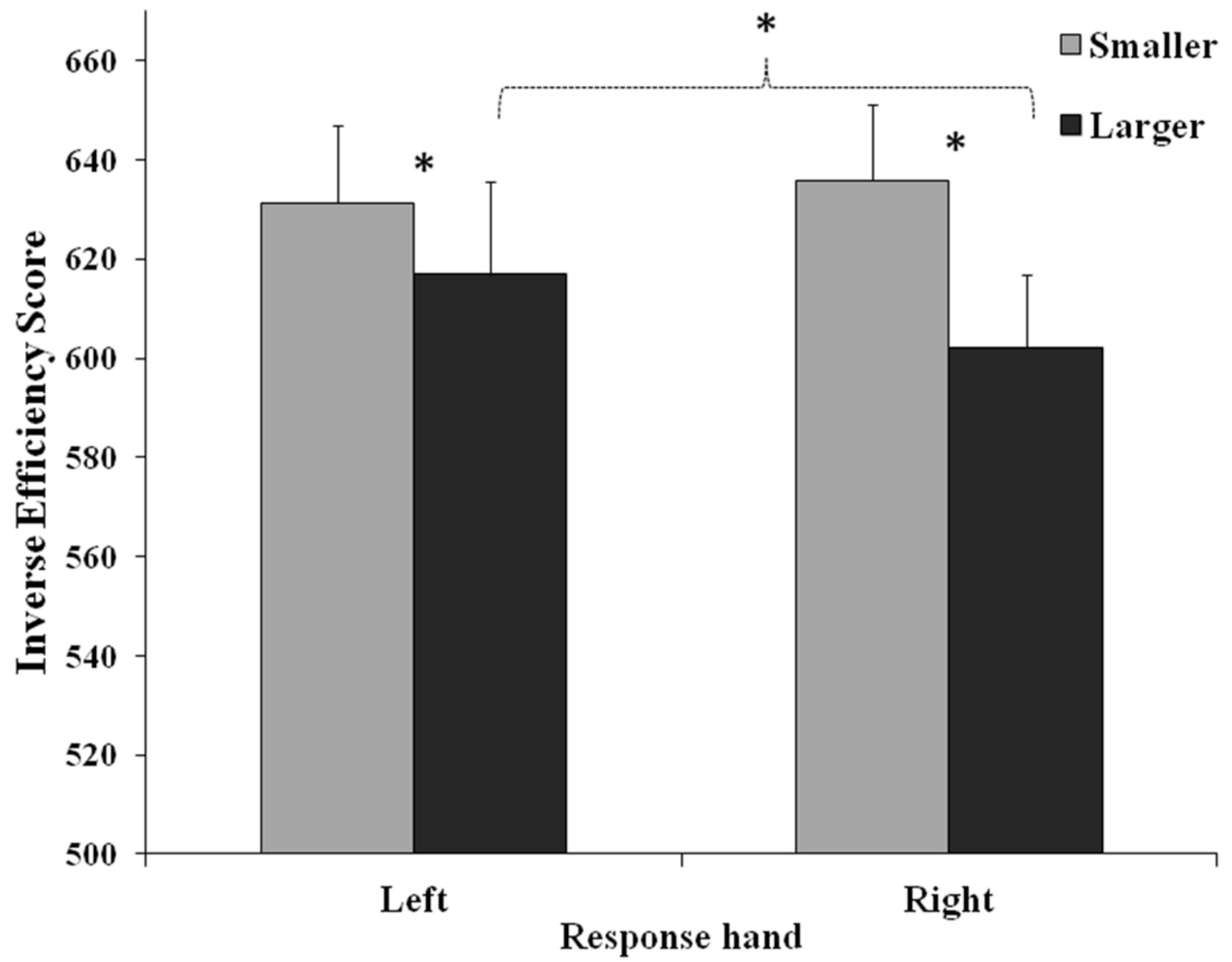




\section{Figure 4}

Interaction among Depicted hand, Response hand and Number

Interaction among Depicted hand, Response hand and Number: the graph shows the results obtained in two separate ANOVAs carried out for Palm-down images (panel A, no significant interaction) and Palm-up images (panel B, significant interaction), separately. Bars represent standard errors. Asterisks show the significant comparisons.
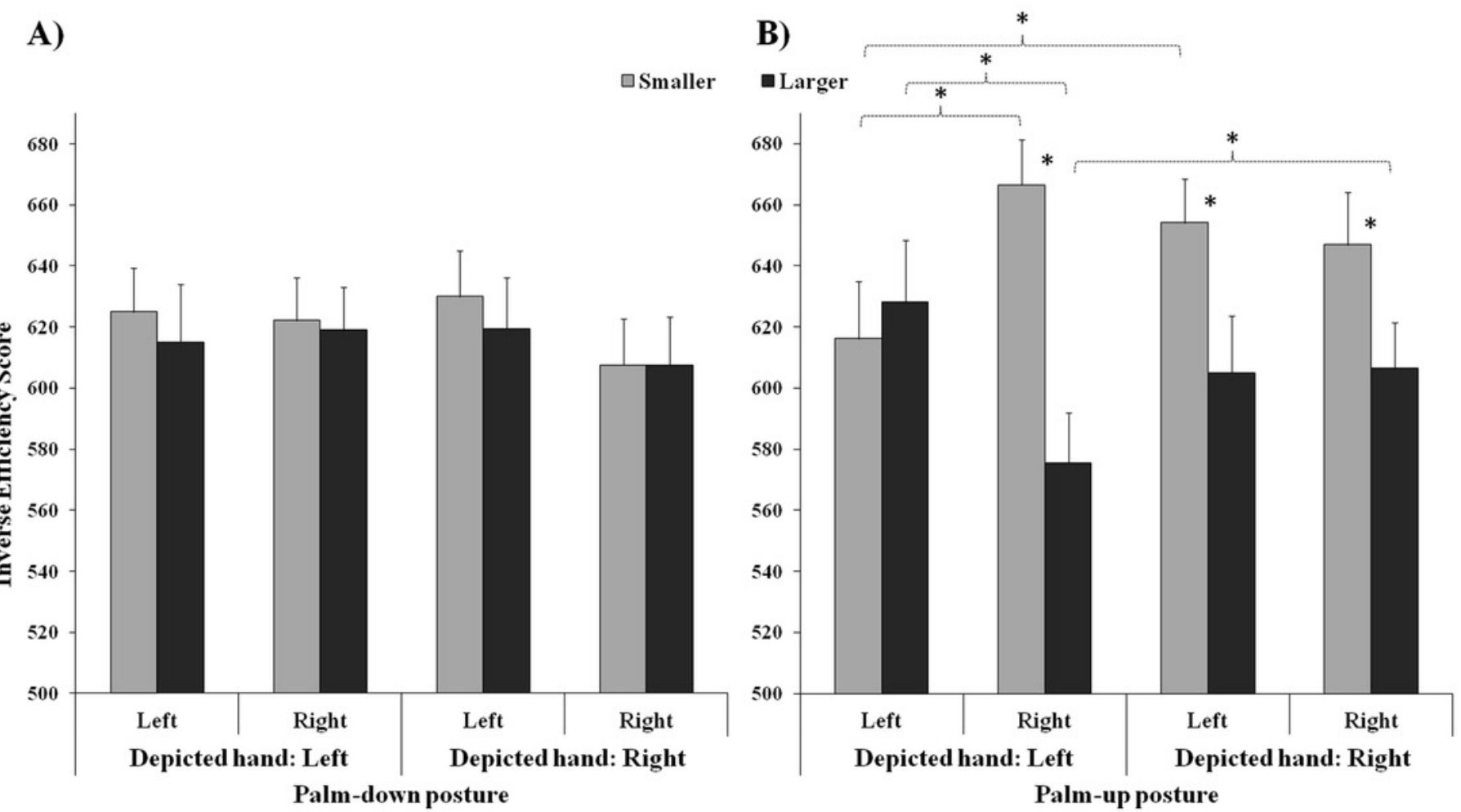

Palm-down posture Palm-up posture 


\section{Figure 5}

Performance of participants in Experiment 2

Performance of participants in Experiment 2: the gray columns show the performance with hand images (bars represent standard errors). Boxes in the first row represent the SNARCcongruent conditions (left: smaller magnitudes categorized with the left hand; right: larger magnitudes categorized with the right hand), boxes in the second row represent the SNARCincongruent conditions (left: larger magnitudes categorized with the left hand; right: smaller magnitudes categorized with the right hand). The horizontal lines in each box represent the mean performance with Arabic code numbers (continuous line shows the significant difference, dotted line shows the non-significant difference between Arabic code and finger code). 

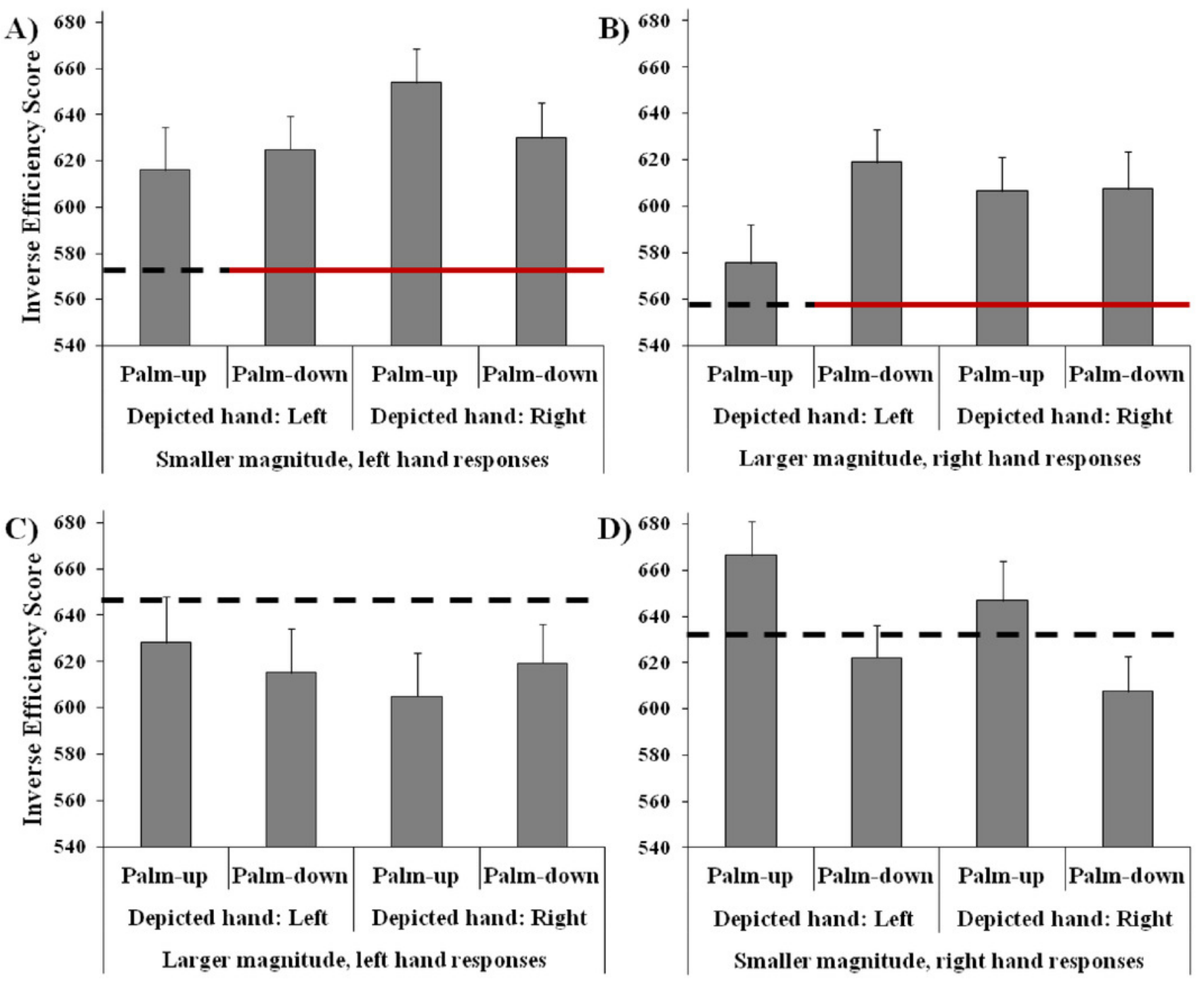


\section{Table $\mathbf{1}$ (on next page)}

Pattern of finger counting from 1 to 10 in Experiment 1

Pattern of finger counting from 1 to 10 in Experiment 1: frequencies (column $\mathrm{N}$ ) and percentages (column \%). The first column indicates the hand posture: UP for "palm-up", DW for "palm-down". Letter pairs represent the hand (L for "left"; R for "right") and the finger used to indicate the number at the top of the column (T: thumb, I: index, M: middle, R: ring, P: pinkie). 


\section{Table 1:}

2

\begin{tabular}{|c|c|c|c|c|c|c|c|c|c|c|c|c|}
\hline & 1 & 2 & 3 & 4 & 5 & 6 & 7 & 8 & 9 & 10 & $\mathbf{N}$ & $\%$ \\
\hline UP & LT & $\mathrm{LI}$ & LM & LR & LP & RT & $\mathrm{RI}$ & $\mathrm{RM}$ & $\mathrm{RR}$ & $\mathrm{RP}$ & 52 & 19.40 \\
\hline DW & $\mathrm{LT}$ & $\mathrm{LI}$ & LM & LR & LP & RT & $\mathrm{RI}$ & RM & $\mathrm{RR}$ & $\mathrm{RP}$ & 1 & 0.373 \\
\hline UP & $\mathrm{LT}$ & $\mathrm{LI}$ & LM & LR & LP & RP & $\mathrm{RR}$ & RM & $\mathrm{RI}$ & RT & 3 & 1.119 \\
\hline UP & $\mathrm{LT}$ & $\mathrm{LI}$ & LM & LR & LP & LT & $\mathrm{LI}$ & LM & LR & LP & 0 & 0 \\
\hline UP & $\mathrm{RT}$ & $\mathrm{RI}$ & $\mathrm{RM}$ & $\mathrm{RR}$ & $\mathrm{RP}$ & LT & $\mathrm{LI}$ & LM & LR & LP & 200 & 74.63 \\
\hline DW & $\mathrm{RT}$ & $\mathrm{RI}$ & $\mathrm{RM}$ & $\mathrm{RR}$ & $\mathrm{RP}$ & LT & $\mathrm{LI}$ & LM & LR & LP & 3 & 1.119 \\
\hline UP & $\mathrm{RT}$ & $\mathrm{RI}$ & $\mathrm{RM}$ & $\mathrm{RR}$ & $\mathrm{RP}$ & LP & LR & LM & $\mathrm{LI}$ & LT & 2 & 0.746 \\
\hline UP & $\mathrm{RT}$ & $\mathrm{RI}$ & $\mathrm{RM}$ & RR & $\mathrm{RP}$ & RT & $\mathrm{RI}$ & RM & $\mathrm{RR}$ & $\mathrm{RP}$ & 7 & 2.612 \\
\hline & & & & & & & & & & & 268 & 100 \\
\hline
\end{tabular}

3

4 